\title{
GENUS 2 FIELDS WITH DEGREE 3 ELLIPTIC SUBFIELDS
}

\author{
T. SHASKA
}

\begin{abstract}
In this paper we study genus 2 function fields $K$ with degree 3 elliptic subfields. We show that the number of $A u t(K)$-classes of such subfields of fixed $K$ is $0,1,2$ or 4 . Also we compute an equation for the locus of such $K$ in the moduli space of genus 2 curves.
\end{abstract}

\section{INTRODUCTION}

We study genus two curves $\mathcal{C}$ whose function fields have a degree 3 subfield of genus 1. Such subfields we call elliptic subfields. Such curves $\mathcal{C}$ have already occurred in the work of Hermite, Goursat, Burkhardt, Brioschi, and Bolza, see Krazer [6] (p. 479). More generally, degree $n$ elliptic subfields of genus 2 fields have been studied by Frey [1], Frey and Kani [2], Kuhn [8], Shaska [11], Shaska and Voelklein 13. In the degree 3 case, explicit equations can be used to answer questions which remain open in the general case. This was the theme of the author's $\mathrm{PhD}$ thesis (see [12]) which also covered the case of degree 2 (which is subsumed in $[13])$.

Equation (5) gives a normal form for pairs $(K, E)$ where $E$ is a degree 3 elliptic subfield of $K$. This normal form depends on two parameters $a, b \in k$. Isomorphism classes of pairs $(K, E)$ are parameterized by parameters $u, v$ where $u:=a b$ and $v:=b^{3}$. This yields an expression for the invariants $\left(i_{1}, i_{2}, i_{3}\right)$ of $K$ (see Igusa [9]) in terms of $u$ and $v$. Our central object of study is the map

$$
\theta:(u, v) \rightarrow\left(i_{1}, i_{2}, i_{3}\right)
$$

This map is shown to have degree 2 , which means that a general genus 2 field having elliptic subfields of degree 3 has exactly two such subfields. There are exactly four genus 2 fields which have four Aut $(K)$-classes and a 1-dimensional family of such fields with one $\operatorname{Aut}(K)$-class of such subfields.

Although the map $\theta$ is given by explicit equations (see (13)), it is a non-trivial computational task to show that it has degree 2 and to compute the action of its Galois group on $u$ and $v$. The direct approach exceeds available computer power, so we introduce auxiliary parameters $r_{1}, r_{2}$ that parameterize pairs of cubic polynomials. They arise from the fact that the subfield $E$ induces a particular sextic defining $K$ which splits naturally as a product of 2 cubic polynomials. We show that $\theta$ factorizes as

$$
(u, v) \rightarrow\left(r_{1}, r_{2}\right) \rightarrow\left(i_{1}, i_{2}, i_{3}\right)
$$

where the latter map is birational, and the former has degree 2. Thus $r_{1}, r_{2}$ yield a birational parameterization of the locus $\mathcal{L}_{3}$ of genus 2 fields having a degree 3 elliptic subfield. That $\mathcal{L}_{3}$ is a rational variety follows also from the general theory of "diagonal modular surfaces", see Kani 泊. We also compute the equation in $i_{1}, i_{2}, i_{3}$ that defines $\mathcal{L}_{3}$ as a sublocus of the moduli space $\mathcal{M}_{2}$ of genus 2 curves. 
We further find relations between the j-invariants of the degree 3 elliptic subfields of $K$ and classify all $K$ in $\mathcal{L}_{3}$ with extra automorphisms.

All the computations were done using Maple [10].

Acknowledgment: I would like to express my sincere gratitude to my $\mathrm{PhD}$ advisor Prof. H. Voelklein for all the time and effort spent in guiding me towards my dissertation (from which this paper originated).

\section{Genus Two Fields With Degree 3 Elliptic Subfields}

Let $k$ be an algebraically closed field of characteristic 0. All function fields will be over $k$. Let $K$ be a genus 2 function field and $E$ a degree 3 elliptic subfield of $K$.

The associated extension $k(X) / k(U)$. It is well-known that $K$ has exactly one genus zero subfield of degree 2 , which we denote by $k(X)$. The generator of $\operatorname{Gal}(K / k(X))$ is the hyperelliptic involution of $K$. It fixes each elliptic subfield of $K$, see Tamme 114. Hence the field $E \cap k(X)$, denote it by $k(U)$, is a subfield of $E$ of degree 2 .

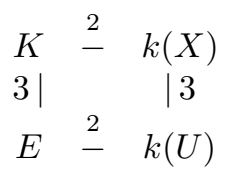

Ramification of $K / E$. Either $K / E$ is ramified at exactly 2 places of $K$, of ramification index 2 , or at one place of $K$, of ramification index 3 . It follows immediately from the Riemann-Hurwitz formula. The former (resp., latter) case we call the non-degenerate (resp., degenerate) case, as in [1].

Definition 1. A non-degenerate pair (resp., degenerate pair) is a pair $(K, E)$ such that $K$ is a genus 2 field with a degree 3 elliptic subfield $E$ where the extension $K / E$ is ramified in two (resp., one) places. Two such pairs $(K, E)$ and $\left(K^{\prime}, E^{\prime}\right)$ are called isomorphic if there is a $k$-isomorphism $K \rightarrow K^{\prime}$ mapping $E \rightarrow E^{\prime}$.

Ramification of $k(X) / k(U)$. In the non-degenerate (resp., degenerate ) case $k(X) / k(U)$ is ramified at exactly 4 (resp., 3) places of $k(X)$ each of ramification index 2 (resp., one of index 3 and the other two of index 2), see [1].

Invariants of $K$. We denote by $J_{2}, J_{4}, J_{6}, J_{10}$ the classical invariants of $K$, for their definitions see [9] or [13]. These are homogeneous polynomials (of degree indicated by subscript) in the coefficients of a sectix $f(X, Z)$ defining $K$

$$
Y^{2}=f(X, Z)=a_{6} X^{6}+a_{5} X^{5} Z+\cdots+a_{1} X Z^{5}+a_{0}
$$

and they are a complete set of $S L_{2}(k)$-invariants (acting by coordinate change). The absolute invariants

$$
i_{1}:=144 \frac{J_{4}}{J_{2}^{2}}, \quad i_{2}:=-1728 \frac{J_{2} J_{4}-3 J_{6}}{J_{2}^{3}}, \quad i_{3}:=486 \frac{J_{10}}{J_{2}^{5}}
$$

are even $G L_{2}(k)$-invariants. Two genus 2 curves with $J_{2} \neq 0$ are isomorphic if and only if they have the same absolute invariants.

Main Theorem: Let $K$ be a genus 2 field and $e_{3}(K)$ the number of Aut $(K / k)$ classes of elliptic subfields of $K$ of degree 3. Then; 
i) $e_{3}(K)=0,1,2$, or 4

ii) $e_{3}(K) \geq 1$ if and only if the classical invariants of $K$ satisfy the irreducible equation $F\left(J_{2}, J_{4}, J_{6}, J_{10}\right)=0$ displayed in Appendix $A$.

There are exactly two genus 2 curves (up to isomorphism) with $e_{3}(K)=4$, see 4.2. The case $e_{3}(K)=1$ (resp., 2) occurs for a 1-dimensional (resp., 2-dimensional) family of genus 2 curves, see section 4 .

2.1. The non-degenerate case. Let $(K, E)$ be a non-degenerate pair and $k(X)$ and $k(U)$ their associated genus 0 subfields. Both $k(X) / k(U)$ and $E / k(U)$ are ramified at 4 places of $k(U)$, three of which are in common. Take the common places to be $U=q_{1}, q_{2}, q_{3}$. Also, take $U=0$ (resp., $U=\infty$ ) the place ramified in $k(X)$ but not in $E$ (resp., in $E$ but not $k(X)$ ). Take $X=0$ (resp., $X=\infty$ ) the place over $U=0$ of ramification index 2 (resp., 1). In the following figure bullets (resp., circles) represent places of ramification index 2 (resp., 1).

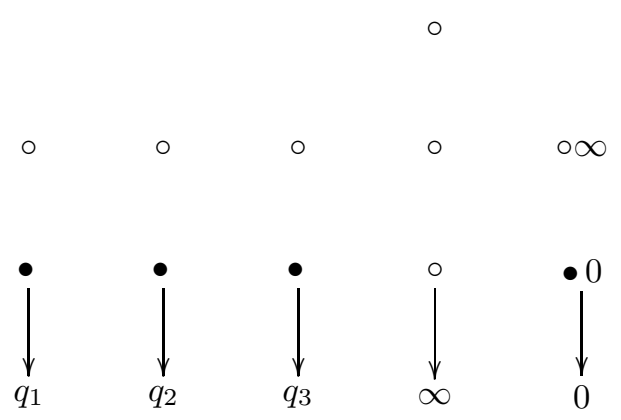

FiguRE 1. Ramification of $k(X) / k(U)$

Thus, $U=\phi(X)=l \frac{X^{2}}{X^{3}+a X^{2}+b X+c}$, where $l, a, b, c \in k$ such that $X^{3}+a X^{2}+$ $b X+c$ has no multiple roots and $l, c \neq 0$. We can make $l=1$ and $c=1$ by replacing $U$ and $X$ by scalar multiples. Then,

$$
U=\phi(X)=\frac{X^{2}}{X^{3}+a X^{2}+b X+1}
$$

Remark 2. These conditions determine $X$ up to multiplication by a third root of unity $\xi_{3} \in k$. Replacing $X$ by $\xi_{3} X$ replaces $(a, b)$ by $\left(\xi_{3}^{2} a, \xi_{3} b\right)$. Invariants of this transformation are:

$$
\begin{aligned}
& u=a b \\
& v=b^{3}
\end{aligned}
$$

The derivative of $\phi(X)$ is

$$
\phi^{\prime}(X)=-\frac{X\left(X^{3}-b X-2\right)}{\left(X^{3}+a X^{2}+b X+1\right)^{2}}
$$

Taking the resultant with respect to $z$ of $z^{2}-b z-2$ and $\frac{\phi(x)-\phi(z)}{x-z}$ we get

$$
\left(x^{3}-b x-2\right)\left(4 x^{3}+b^{2} x^{2}+2 b x+1\right)
$$


The roots of this polynomial correspond to the 6 places of $k(X)$ over the places $U=q_{1}, q_{2}, q_{3}$. Thus, the 6 places of $k(X)$ ramified in $K$ correspond to the roots of the polynomial on the right side of

$$
Y^{2}=\left(X^{3}+a X^{2}+b X+1\right)\left(4 X^{3}+b^{2} X^{2}+2 b X+1\right)
$$

which gives an equation of $K$. The discriminant of the sextic is nonzero, hence

$$
\Delta:=\left(4 a^{3}+27-18 a b-a^{2} b^{2}+4 b^{3}\right)^{2}\left(b^{3}-27\right) \neq 0
$$

One checks that the element

$$
V=Y \frac{X^{3}-b X-2}{F(X)^{2}}
$$

satisfies

$$
V^{2}=\left(U-q_{1}\right)\left(U-q_{2}\right)\left(U-q_{3}\right)=U^{3}+2 \frac{a b^{2}-6 a^{2}+9 b}{R} U^{2}+\frac{12 a-b^{2}}{R} U-\frac{4}{R}
$$

where $R:=\frac{\Delta}{\left(b^{3}-27\right)}$. Thus, $E=k(U, V)$.

Lemma 3. For $(a, b) \in k^{2}$ with $\Delta \neq 0$, equation (5) defines a genus 2 field $K_{a, b}=$ $k(X, Y)$. It has a non-degenerate degree 3 elliptic subfield $E_{a, b}=k(U, V)$, where $U$ and $V$ are given in (2) and (7). Two such pairs $\left(K_{a, b}, E_{a, b}\right)$ and $\left(K_{a^{\prime}, b^{\prime}}, E_{a^{\prime}, b^{\prime}}\right)$ are isomorphic if and only if $u=u^{\prime}$ and $v=v^{\prime}$ (where $u, v$ and $u^{\prime}, v^{\prime}$ are associated with $a, b$ and $a^{\prime}, b^{\prime}$, respectively, by (3)).

Proof. The first two statements follow by reversing the above arguments. If $u=u^{\prime}$, $v=v^{\prime}$ then $a^{\prime}=\xi_{3}^{i} a, b^{\prime}=\xi_{3}^{i} b$ for some $i$. Then clearly the two associated nondegenerate pairs are isomorphic. The converse follows from Remark 2 .

Proposition 4. The $(u, v) \in k^{2}$ with $\Delta \neq 0$ bijectively parameterize the isomorphism classes of non-degenerate pairs $(K, E)$ (via the parameterization defined in Lemma 3).

Proof. The proof follows from Lemma 3.

From the normal form of $K$ in (5) we can compute the classical invariants $J_{2}, J_{4}, J_{6}, J_{10}$ in terms of $u, v$. These expressions satisfy equation (33) which proves one implication of claim ii) of the theorem for the non-degenerate pairs. In section 4 we explain how equation (33) was found.

2.2. The degenerate case. Let $K / E$ be a degenerate pair. Then $k(X) / k(U)$ is ramified at exactly three places of $k(U)$ which are also ramified in $E$. Let $U=0$ be the place ramified in $E$ but not in $k(X)$. Take $U=\infty$ be the place of $k(U)$ that is totally ramified in $k(X)$ and $X=\infty$ the place over it.

Then, $U=l\left(X^{3}+a X^{2}+b X+c\right)$ for $a, b, c, l \in k$ such that $X^{3}+a X^{2}+b X+c$ has no multiple roots and $l \neq 0$. Replacing $X$ by $X+\frac{a}{3}$ and $U$ by a scalar multiple we get $U=X^{3}+b X+c$. Note that $b \neq 0$ (otherwise two of the places of $k(U)$ ramified in $k(X)$ coalesce). Thus, by replacing $X$ by a scalar multiple we can also get $b=1$. Then,

$$
U=X^{3}+X+c
$$

As in Lemma 3 we get $K=k(X, Y)$ such that

$$
Y^{2}=\left(3 X^{2}+4\right)\left(X^{3}+X+c\right)
$$




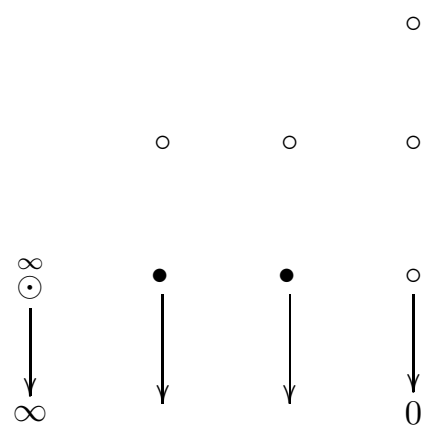

Figure 2. Ramification of $k(X) / k(U)$, degenerate case.

where $c^{2} \neq-\frac{4}{27}$. Then, $V=Y\left(3 X^{2}+1\right)$ satisfies the equation

$$
V^{2}=U\left(27 U^{2}-54 c U+4+27 c^{2}\right)
$$

Thus, $E=k(U, V)$.

Remark 5. In this case, those $w:=c^{2}$ with $w \neq-\frac{4}{27}$ bijectively parameterize the isomorphism classes of degenerate pairs $K / E$. The proof is analogous to the non-degenerate case.

Since

$$
\begin{aligned}
J_{2} & =774 \\
J_{4} & =36(268+837 w) \\
J_{6} & =36\left(76760+290574 w-729 w^{2}\right) \\
J_{10} & =432(27 w+4)
\end{aligned}
$$

the map

$$
\begin{gathered}
k \backslash\left\{-\frac{4}{27}\right\} \rightarrow k^{3} \\
w \rightarrow\left(i_{1}, i_{2}, i_{3}\right)
\end{gathered}
$$

is injective. Thus, a genus 2 field $K$ has at most one $A u t(K)$-orbit of subfields $E$ such that $(K, E)$ is a degenerate pair. One can check that $J_{2}, J_{4}, J_{6}, J_{10}$ satisfy equation (33). This completes the proof that the condition in part (ii) of Main Theorem is necessary.

¿From (10) we compute that the locus of those $K$ being part of a degenerate pair $(K, E)$ is given by

$$
\begin{aligned}
& 3418801 i_{1}^{2}-2550732480 i_{1}+4023336960+611249816 i_{2}=0 \\
& 161446939560824832 i_{3}+23630752512 i_{1}^{2}-6321363049 i_{1}^{3}-29445783552 i_{1} \\
& +12230590464=0
\end{aligned}
$$

(Note $J_{2}=774 \neq 0$ on this locus, so $i_{1}, i_{2}, i_{3}$ are everywhere defined). For later use we note the following: If $\left(K, E_{0}\right)$ is a non-degenerate pair with parameters $u, v$ then there exists a degenerate pair $\left(K, E_{1}\right)$ (for same $K$ ) if and only if

$$
2 v-9 u+27=0
$$


This is obtained by expressing $i_{1}, i_{2}, i_{3}$ in (11) in terms of $u, v$.

\section{FunCtion Field of $\mathcal{L}_{3}$}

The absolute invariants $i_{1}, i_{2}, i_{3}$ in terms of $u, v$ are

$$
\begin{aligned}
i_{1}= & \frac{144}{v\left(-405+252 u+4 u^{2}-54 v-12 u v+3 v^{2}\right)^{2}}\left(1188 u^{3}-8424 u v+u^{4} v-24 u^{4}\right. \\
& \left.+14580 v-66 u^{3} v+138 u v^{2}+297 u^{2} v+945 v^{2}-36 v^{3}+9 u^{2} v^{2}\right) \\
i_{2}= & -\frac{864}{v^{2}\left(-405+252 u+4 u^{2}-54 v-12 u v+3 v^{2}\right)^{3}}\left(-81 v^{3} u^{4}+2 u^{6} v^{2}+234 u^{5} v^{2}\right. \\
& +3162402 u v^{2}-21384 v^{3} u+26676 v^{4}-473121 v^{3}-72 u^{6} v-5832 v^{4} u+14850 v^{3} u^{2} \\
& -72 v^{3} u^{3}+324 v^{4} u^{2}-650268 u^{3} v-5940 u^{3} v^{2}-3346110 v^{2}+432 u^{6}-1350 u^{4} v^{2} \\
& +136080 u^{4} v-7020 u^{5} v-307638 u^{2} v^{2} \\
i_{3}= & -243 \frac{(v-27)\left(4 u^{3}-u^{2} v-18 u v+4 v^{2}+27 v\right)^{3}}{v^{3}\left(-405+252 u+4 u^{2}-54 v-12 u v+3 v^{2}\right)^{5}}
\end{aligned}
$$

We will sometimes view $u, v$ as parameters from $k$ and sometimes as the corresponding coordinate functions on $k^{2}$. From the context it will be clear which point of view we are taking.

Lemma 6. $\left[k\left(i_{1}, i_{2}, i_{3}\right): k(u, v)\right] \leq 2$

Proof. ¿From the resultants of equations in (13) we determine that $\left[k(v): k\left(i_{1}, i_{2}\right)\right]=$ $16,\left[k(v): k\left(i_{2}, i_{3}\right)\right]=40$, and $\left[k(v): k\left(i_{1}, i_{3}\right)\right]=26$. We can show that $u \in$ $k\left(i_{1}, i_{2}, i_{3}, v\right)$, the expression is large and we don't display it (the interested reader can check 12]). Since $\left[k(u, v): k\left(i_{1}, i_{2}, i_{3}\right)\right]$ must be a common divisor of 16,26 , and 40 , then the claim follows.

We need to show that $\left[k(u, v): k\left(i_{1}, i_{2}, i_{3}\right)\right]=2$. Since we know that $[k(u, v)$ : $\left.k\left(i_{1}, i_{3}\right)\right]=26$, this is equivalent to showing that $\left[k\left(i_{1}, i_{2}, i_{3}\right): k\left(i_{1}, i_{3}\right)\right]=13$. This follows once we have equation (33) in terms of $i_{1}, i_{2}, i_{3}$ but at this stage we cannot derive it: Eliminating $u, v$ from equations (13) exceeds available computer power. We use additional invariants $r_{1}, r_{2}$ to overcome this problem.

3.1. Invariants of Two Cubics. We define the following invariants of two cubic polynomials. For $F(X)=a_{3} X^{3}+a_{2} X^{2}+a_{1} X+a_{0}$ and $G(X)=b_{3} X^{3}+b_{2} X^{2}+$ $b_{1} X+b_{0}$ define

$$
H(F, G):=a_{3} b_{0}-\frac{1}{3} a_{2} b_{1}+\frac{1}{3} a_{1} b_{2}-a_{0} b_{3}
$$

We denote by $R(F, G)$ the resultant of $F$ and $G$ and by $D(F)$ the discriminant of F. Also,

$$
r_{1}(F, G)=\frac{H(F, G)^{3}}{R(F, G)}, \quad r_{2}(F, G)=\frac{H(F, G)^{4}}{D(F) D(G)}
$$

Remark 7. In 河 it is shown that $r_{1}, r_{2}$, and $r_{3}=\frac{H(F, G)^{2}}{J_{2}(F G)}$ form a complete system of invariants for unordered pairs of cubics. 
For $F(X)=X^{3}+a X^{2}+b X+1$ and $G(X)=4 X^{3}+b^{2} X^{2}+2 b X+1$ and $u, v$ as in (3) we have

$$
\begin{aligned}
& r_{1}(F, G)=27 \frac{v(v-9-2 u)^{3}}{4 v^{2}-18 u v+27 v-u^{2} v+4 u^{3}} \\
& r_{2}(F, G)=-1296 \frac{v(v-9-2 u)^{4}}{(v-27)\left(4 v^{2}-18 u v+27 v-u^{2} v+4 u^{3}\right)}
\end{aligned}
$$

Taking the resultants from the above equations we get the following equations for $u$ and $v$ over $k\left(r_{1}, r_{2}\right)$ :

$$
\begin{gathered}
65536 r_{1} r_{2}^{3} u^{2}+\left(42467328 r_{2}^{4}+21233664 r_{2}^{4} r_{1}+480 r_{2} r_{1}^{4}+2 r_{1}^{5}+41472 r_{2}^{2} r_{1}^{3}\right. \\
\left.+1548288 r_{2}^{3} r_{1}^{2}-294912 r_{2}^{3} r_{1}\right) u-382205952 r_{2}^{4}+238878720 r_{2}^{4} r_{1}-2654208 r_{2}^{3} r_{1} \\
+13934592 r_{2}^{3} r_{1}^{2}+285696 r_{2}^{2} r_{1}^{3}+2400 r_{2} r_{1}^{4}+7 r_{1}^{5}=0 \\
16384 v^{2} r_{2}^{3}+\left(221184 r_{2}^{3} r_{1}+r_{1}^{4}+11520 r_{2}^{2} r_{1}^{2}-442368 r_{2}^{3}+192 r_{2} r_{1}^{3}\right) v \\
-5971968 r_{2}^{3} r_{1}-864 r_{2} r_{1}^{3}-124416 r_{2}^{2} r_{1}^{2}-2 r_{1}^{4}=0
\end{gathered}
$$

Roots of equation (15) (resp., equation (16)) are $u$ and $\beta(u)$ (resp., $v$ and $\beta(v)$ ) where,

$$
\begin{aligned}
& \beta(u)=\frac{(v-3 u)\left(324 u^{2}+15 u^{2} v-378 u v-4 u v^{2}+243 v+72 v^{2}\right)}{(v-27)\left(4 u^{3}+27 v-18 u v-u^{2} v+4 v^{2}\right)} \\
& \beta(v)=-\frac{4(v-3 u)^{3}}{4 u^{3}+27 v-18 u v-u^{2} v+4 v^{2}}
\end{aligned}
$$

It follows that either $\left[k(u, v): k\left(r_{1}, r_{2}\right)\right]=2$ or $k(u, v) / k\left(r_{1}, r_{2}\right)$ is Galois with Klein 4-group as Galois group. In the latter case, the map

$$
\begin{aligned}
\tau: k(u, v) & \rightarrow k(u, v) \\
u & \rightarrow u \\
v & \rightarrow \beta(v)
\end{aligned}
$$

is an involutory automorphism of $k(u, v)$. But by plugging in equation (17) we see that it is not involutory. Thus, $\left[k(u, v): k\left(r_{1}, r_{2}\right)\right]=2$ and $\operatorname{Gal}_{k(u, v) / k\left(r_{1}, r_{2}\right)}=\langle\beta\rangle$.

Lemma 8. The fields $k\left(i_{1}, i_{2}, i_{3}\right)$ and $k\left(r_{1}, r_{2}\right)$ coincide, hence $\left[k(u, v): k\left(i_{1}, i_{2}, i_{3}\right)\right]=$ 2. Moreover;

$$
\begin{aligned}
i_{1} & =\frac{9}{4} \frac{\left(13824 r_{1}^{3} r_{2}^{2}+442368 r_{1}^{2} r_{2}^{3}+5308416 r_{1} r_{2}^{4}+192 r_{1}^{4} r_{2}+r_{1}^{5}+786432 r_{1} r_{2}^{3}+9437184 r_{2}^{4}\right)}{r_{1}\left(-1152 r_{2}^{2}+96 r_{2} r_{1}+r_{1}^{2}\right)^{2}} \\
i_{2} & =\frac{27}{8 r_{1}^{2}\left(-1152 r_{2}^{2}+96 r_{2} r_{1}+r_{1}^{2}\right)^{3}}\left(+79626240 r_{1}^{4} r_{2}^{4}-4076863488 r_{1}^{2} r_{2}^{5}+34560 r_{1}^{6} r_{2}^{2}\right. \\
& +12230590464 r_{1}^{2} r_{2}^{6}+32614907904 r_{1} r_{2}^{6}+14495514624 r_{2}^{6}+288 r_{1}^{7} r_{2}+2211840 r_{1}^{5} r_{2}^{3} \\
& \left.+r_{1}^{8}-212336640 r_{1}^{3} r_{2}^{4}+1528823808 r_{1}^{3} r_{2}^{5}-2359296 r_{1}^{4} r_{2}^{3}\right) \\
i_{3} & =-521838526464 \frac{r_{2}^{9}}{r_{1}^{2}\left(-1152 r_{2}^{2}+96 r_{2} r_{1}+r_{1}^{2}\right)^{5}}
\end{aligned}
$$

Proof. Equations (19) are verified by expressing all variables in terms of $u, v$ (These equations were found by taking suitable resultants). It follows that $k\left(i_{1}, i_{2}, i_{3}\right) \subset$ 
$k\left(r_{1}, r_{2}\right)$. Equality follows since $\left[k(u, v): k\left(r_{1}, r_{2}\right)\right]=2$ and $\left[k(u, v): k\left(i_{1}, i_{2}, i_{3}\right)\right] \leq$ 2 (see Lemma 6).

Remark 9. To find equation (33) we eliminate $r_{1}$ and $r_{2}$ from equations (19). The resulting equation (33) has degree 8, 13, and 20 in $i_{1}, i_{2}, i_{3}$ respectively.

\section{Proof of Main Theorem}

The map

$$
\theta:(u, v) \rightarrow\left(i_{1}, i_{2}, i_{3}\right)
$$

given by (13) has degree 2 , by previous section and it is defined when $J_{2} \neq 0$. For now we assume that $J_{2} \neq 0$ (The case $J_{2}=0$ is treated in section 4.2 ). Denote the minors of the Jacobian matrix of $\theta$ by $M_{1}(u, v), M_{2}(u, v), M_{3}(u, v)$. The solutions of

$$
\left\{\begin{array}{l}
M_{1}(u, v)=0 \\
M_{2}(u, v)=0 \\
M_{3}(u, v)=0
\end{array}\right.
$$

consist of the (non-singular) curve

$$
8 v^{3}+27 v^{2}-54 u v^{2}-u^{2} v^{2}+108 u^{2} v+4 u^{3} v-108 u^{3}=0
$$

\begin{tabular}{|c|c|c|c|}
\hline$(u, v)$ & $\left(i_{1}, i_{2}, i_{3}\right)$ & $\operatorname{Aut}(K)$ & $e_{3}(K)$ \\
\hline$\left(-\frac{7}{2}, 2\right)$ & $\begin{array}{c}J_{10}=0, \quad \text { no associated } \\
\text { genus } 2 \text { field } \mathrm{K}\end{array}$ & & \\
\hline $\begin{array}{c}\left(-\frac{775}{8}, \frac{125}{96}\right) \\
\left(\frac{25}{2}, \frac{250}{9}\right)\end{array}$ & $-\frac{8019}{20},-\frac{1240029}{200}, \frac{531441}{100000}$ & $D_{4}$ & 2 \\
\hline $\begin{array}{l}\left(27-\frac{77}{2} \sqrt{-1}, 23+\frac{77}{9} \sqrt{-1}\right) \\
\left(27+\frac{77}{2} \sqrt{-1}, 23-\frac{77}{9} \sqrt{-1}\right)\end{array}$ & $\left(\frac{729}{2116}, \frac{1240029}{97336}, \frac{531441}{13181630464}\right.$ & $D_{4}$ & 2 \\
\hline $\begin{array}{l}\left(-15+\frac{35}{8} \sqrt{5}, \frac{25}{2}+\frac{35}{6} \sqrt{5}\right) \\
\left(-15-\frac{35}{8} \sqrt{5}, \frac{25}{2}-\frac{35}{6} \sqrt{5}\right)\end{array}$ & $81,-\frac{5103}{25},-\frac{729}{12500}$ & $D_{6}$ & 2 \\
\hline
\end{tabular}

and 7 isolated solutions which we display in table (11) together with the corresponding values $\left(i_{1}, i_{2}, i_{3}\right)$ and properties of the corresponding genus 2 field $K$.

TABLE 1. Exceptional points where $\operatorname{det}(\operatorname{Jac}(\theta))=0$

Let $\bar{u}, \bar{v}, \bar{i}_{1}, \bar{i}_{2}, \bar{i}_{3}$ denote the restrictions of the corresponding functions to the curve (21). Eliminating $u$ from (21) and the defining equation for $i_{1}$ in (13) we get a relation between $v$ and $i_{1}$ which shows $\left[k\left(\bar{v}, \bar{i}_{1}\right): k\left(\bar{i}_{1}\right)\right]=8$; also we get $\bar{u}$ expressed as a rational function of $\bar{v}$ and $\bar{i}_{1}$. Hence $k\left(\bar{v}, \bar{i}_{1}\right)=k(\bar{u}, \bar{v})$ and so $\left[k(\bar{u}, \bar{v}): k\left(\bar{i}_{1}\right)\right]=8$. Similarly we get a relation between $\bar{v}$ and $\bar{i}_{2}$. Eliminating $\bar{v}$ gives

$$
G\left(\bar{i}_{1}, \bar{i}_{2}\right)=0
$$


an irreducible equation between $\bar{i}_{1}$ and $\bar{i}_{2}$ of degree 8 (resp., 12 ) in $\bar{i}_{1}$ (resp., $\bar{i}_{2}$ ). It is fully displayed as equation (34) in Appendix B. Thus, $\left[k\left(\bar{i}_{1}, \bar{i}_{2}\right): k\left(\bar{i}_{1}\right)\right]=8$. Since also $\left[k(\bar{u}, \bar{v}): k\left(\bar{i}_{1}\right)\right]=8$, it follows that $k(\bar{u}, \bar{v})=k\left(\bar{i}_{1}, \bar{i}_{2}\right)$. Hence, $e_{3}(K)=1$ for any $K$ such that the associated $u$ and $v$ satisfy equation (21) except possibly those $(u, v)$ that lie over singular points of the curve (22). We check that all these singular points have multiplicity 2 , hence there are at most two $(u, v)$ points over each of them.

Remark 10. If $K$ has one subfield of degree 3, then it has at least two distinct such subfields. This follows from the explicit construction in [12], Lemma 5.4. (It is actually true for degree $n$ elliptic subfields, for any $n$, see 13 ). Since $e_{3}(K)=1$ for a generic curve $K$ of the locus (22), such $K$ has $\mid$ Aut $(K) \mid>2$. Actually $A u t(K)=V_{4}$. We verified this by using equation (17) from 13 which gives a necessary and sufficient condition for a genus 2 field $K$ to have $\vec{V}_{4} \leq A u t(K)$ (in terms of the classical invariants of $K$ ). Taking the resultant of this equation and the one in Appendix A yields an expression that has (22) (the projectivized version) as a factor. From the conditions given in [13] for $K$ to have Aut $(K)$ strictly bigger than $V_{4}$, one checks that actually $A u t(K)=V_{4}$ for all $K$ in locus (22).

4.1. The general case. By previous section $\theta$ is generically a covering of degree 2 . So there exists a Zariski open subset $\mathcal{U}$ of $k^{2}$ with the following properties: Firstly, $\theta$ is defined everywhere on $\mathcal{U}$ and is a covering of degree 2 from $\mathcal{U}$ to $\theta(\mathcal{U})$. Further, if $\mathfrak{u} \in \mathcal{U}$ then all $\mathfrak{u}^{\prime} \in k^{2}$ with $\theta$ defined at $\mathfrak{u}^{\prime}$ and $\theta\left(\mathfrak{u}^{\prime}\right)=\theta(\mathfrak{u})$ also lie in $\mathcal{U}$. Suppose $\underline{i} \in k^{3}$ such that $\left|\theta^{-1}(\underline{i})\right|>2$ and $\operatorname{det}(\operatorname{Jac}(\theta))$ does not vanish at any point of $\theta^{-1}(\underline{i})$. Then by implicit function theorem, there is an open ball $B$ around each element of $\theta^{-1}(\underline{i})$ such that each point in $\theta(B)$ has $>2$ inverse images under $\theta$. But $B$ has to intersect the Zariski open set $\mathcal{U}$. This is a contradiction. Thus, if $\underline{i} \in k^{3}$ and $\left|\theta^{-1}(\underline{i})\right|>2$, then $\operatorname{det}(\operatorname{Jac}(\theta))=0$ at some point of $\theta^{-1}(\underline{i})$ and so $\underline{i}$ satisfies $(22)$. It follows that if $K$ is a genus 2 field with $J_{2} \neq 0$ and $G \neq 0$ then $e_{3}(K)=2$.

Note that if there is a degenerate pair $\left(K, E_{1}\right)$ and a non-degenerate pair $\left(K, E_{2}\right)$ (for the same $K)$ then for the $(u, v)$-invariants of the latter we have (12), hence $\beta(v)=27$ and so the $\beta$-image of $(u, v)$ doesn't correspond to a non-degenerate pair.

We can now complete the proof of part (ii) of Main Theorem. We noted before that the condition in (ii) is necessary. This condition (see Appendix A) defines an irreducible sublocus of $\mathcal{M}_{2}$ because the corresponding equation is irreducible (by Maple). The locus $e_{3}(K) \geq 1$ lies in this sublocus and contain a dense subset of it (image of $\theta$ ). By Lange [3], the locus $e_{3}(K) \geq 1$ is Zariski closed, hence equals the locus defined by the equation in Appendix A.

4.2. Exceptional Cases for $J_{2}=0$. We consider the case when $J_{2}=0$, where

$$
\begin{aligned}
J_{2} & =-2\left(3 v^{2}+4 u^{2}-12 u v+252 u-54 v-405\right) \\
& =\frac{2}{3}(3 v-6 u+2 u \sqrt{6}-27-18 \sqrt{6})(3 v-27+18 \sqrt{6}-6 u-2 u \sqrt{6})
\end{aligned}
$$

Genus 2 fields $K$ with $J_{2}=J_{4}=0$ and $J_{6} \neq 0$ (resp., $J_{2}=J_{6}=0$ and $J_{4} \neq 0$ ) are classified up to isomorphism by the invariant $\frac{J_{6}^{5}}{J_{10}^{3}}$ (resp., $\left.\frac{J_{4}^{5}}{J_{10}^{2}}\right)$, see Igusa [9]. In the first (resp., second) case there are exactly two (resp., four) such $K$ with $e_{3}(K) \geq 1$ and they all have $e_{3}(K)=2$. 
The invariants

$$
a_{1}:=\frac{J_{4} \cdot J_{6}}{J_{10}}, \quad a_{2}:=\frac{J_{6} \cdot J_{10}}{J_{4}^{4}}
$$

determine genus two fields with $J_{2}=0, J_{4} \neq 0$, and $J_{6} \neq 0$ up to isomorphism, see Igusa [9]. A field $K$ with such invariants has $e_{3}(K) \geq 1$ if and only if

(25)

$46656 a_{1}^{5} a_{2}^{3}+7558272 a_{1}^{4} a_{2}^{3}+1259712 \sqrt{6} a_{1}^{4} a_{2}^{3}-15552 a_{1}^{4} a_{2}^{2}-12427478784 a_{1}^{3} a_{2}^{2}+4917635712 \sqrt{6} a_{1}^{3} a_{2}^{2}$ $+1728 a_{1}^{3} a_{2}-656217531654480 a_{1}^{2} a_{2}^{2}+267571209034080 \sqrt{6} a_{1}^{2} a_{2}^{2}-1844125056 a_{1}^{2} a_{2}+743525568 \sqrt{6} a_{1}^{2} a_{2}$ $-64 a_{1}^{2}-6334497449472117312 a_{1} a_{2}^{2}+2585860435265558832 \sqrt{6} a_{1} a_{2}^{2}+230833239838992 a_{2} a_{1}-$ $94237227087840 a_{2} a_{1} \sqrt{6}-601244429975805030777 a_{2}^{2}+245429539257764380572 a_{2}^{2} \sqrt{6}=0$

or (26)

$$
\begin{gathered}
230833239838992 a_{2} a_{1}+94237227087840 a_{2} a_{1} \sqrt{6}-12427478784 a_{1}^{3} a_{2}^{2}-4917635712 \sqrt{6} a_{1}^{3} a_{2}^{2} \\
+1728 a_{1}^{3} a_{2}-15552 a_{1}^{4} a_{2}^{2}-656217531654480 a_{1}^{2} a_{2}^{2}-267571209034080 \sqrt{6} a_{1}^{2} a_{2}^{2}+46656 a_{1}^{5} a_{2}^{3}-64 a_{1}^{2} \\
-601244429975805030777 a_{2}^{2}-245429539257764380572 a_{2}^{2} \sqrt{6}-1844125056 a_{1}^{2} a_{2}-743525568 \sqrt{6} a_{1}^{2} a_{2} \\
-6334497449472117312 a_{1} a_{2}^{2}-2585860435265558832 \sqrt{6} a_{1} a_{2}^{2}+7558272 a_{1}^{4} a_{2}^{3}-1259712 \sqrt{6} a_{1}^{4} a_{2}^{3}=0
\end{gathered}
$$

As one can check by discussing the map

$$
\vartheta:(u, v) \rightarrow\left(a_{1}, a_{2}\right)
$$

these fields $K$ generically have $e_{3}(K)=2$. It remains to check the singular points of the curves (25) and (26). The intersection of the two components is empty. Each component has four singular points two of which have $e_{3}(K)=2$ and the other two have $e_{3}(K)=4$. Thus there are exactly four genus 2 fields with $e_{3}(K)=4$. Two of them satisfy (25), we call them $P_{1}$ and $P_{2}$. The other two are obtained from $P_{1}, P_{2}$ by applying the automorphism $\sqrt{6} \rightarrow-\sqrt{6}$.

$$
\begin{array}{|c|c|}
\hline P_{1} & a_{1}=-\frac{77169}{8}+\frac{30759}{8} \sqrt{6}, \quad a_{2}=\frac{13783592}{23149125}+\frac{5629912}{23149125} \sqrt{6} \\
\hline P_{2} & a_{1}=-\frac{650835}{8}+\frac{268785}{8} \sqrt{6}, \quad a_{2}=-\frac{2984}{20002028625}-\frac{144872}{60006085875} \sqrt{6} \\
\hline
\end{array}
$$

TABLE 2. Singular points of (25) with $e_{3}(K)=4$

The four values of $(u, v)$ corresponding to $P_{1}$ (resp., $\left.P_{2}\right)$ are obtained as follows: $u$ is determined by $v$ via

$$
u=\frac{1}{2}(3+\sqrt{6})(v-9-6 \sqrt{6})
$$

and $v$ is one of the 4 roots of

$$
\begin{aligned}
& 9 v^{4}+(-693-27 \sqrt{6}) v^{3}+(15141+1749 \sqrt{6}) v^{2}+(-66414-33396 \sqrt{6}) v \\
&-1368 \sqrt{6}+3388=0
\end{aligned}
$$

(resp.)

$$
\begin{aligned}
3 v^{4}+(-153+33 \sqrt{6}) v^{3}+(3129-1219 \sqrt{6}) v^{2}+ & (-10854-11556 \sqrt{6}) v \\
& +176904 \sqrt{6}+450036=0
\end{aligned}
$$


This completes the proof of the Main Theorem.

\section{J-INVARIANTS}

A genus 2 field $K$ corresponding to a generic point of locus $e_{3}(K) \geq 1$ has exactly 2 elliptic subfields $E_{1}$ and $E_{2}$ of degree 3 . We can take $E_{1}=E$ from lemma 3. Its j-invariant is

$$
j_{1}=16 v \frac{\left(v u^{2}+216 u^{2}-126 v u-972 u+12 v^{2}+405 v\right)^{3}}{(v-27)^{3}\left(4 v^{2}+27 v+4 u^{3}-18 v u-v u^{2}\right)^{2}}
$$

The automorphism $\beta \in \operatorname{Gal}_{k(u, v) / k\left(r_{1}, r_{2}\right)}$ permutes $E_{1}$ and $E_{2}$, therefore switches $j_{1}$ and $j_{2}$. Then $j_{2}=\beta\left(j_{1}\right)$ is

$$
j_{2}=-256 \frac{\left(u^{2}-3 v\right)^{3}}{v\left(4 v^{2}+27 v+4 u^{3}-18 v u-v u^{2}\right)}
$$

For the equation of $E_{2}$ using a different approach see [12], chapter 5 .

5.1. Isomorphic Elliptic Subfields. Suppose that $E_{1} \cong E_{2}$. Then, $j_{1}=j_{2}$ implies that

$$
8 v^{3}+27 v^{2}-54 u v^{2}-u^{2} v^{2}+108 u^{2} v+4 u^{3} v-108 u^{3}=0
$$

or

$$
\begin{aligned}
& 324 v^{4} u^{2}-5832 v^{4} u+37908 v^{4}-314928 v^{3} u-81 v^{3} u^{4}+255879 v^{3}+30618 v^{3} u^{2} \\
& -864 v^{3} u^{3}-6377292 u v^{2}+8503056 v^{2}-324 u^{5} v^{2}+2125764 u^{2} v^{2}-215784 u^{3} v^{2} \\
& +14580 u^{4} v^{2}+16 u^{6} v^{2}+78732 u^{3} v+8748 u^{5} v-864 u^{6} v-157464 u^{4} v+11664 u^{6}=0
\end{aligned}
$$

Remark 11. The former equation is the condition that $\operatorname{det}(\operatorname{Jac}(\theta))=0$ see equation (21).

Remark 12. If $e_{3}(K) \geq 1$ then the automorphism group Aut $(K / k)$ is one of the following: $\mathbb{Z}_{2}, V_{4}, D_{8}$, or $D_{12}$. $\mathbb{Z}_{2}$ is the generic case, $V_{4}$ is a 1-dimensional family. There are exactly 6 genus 2 fields with automorphism group $D_{8}$ (resp., $D_{12}$ ).

For the proof see [12].

\section{Appendix A:}

Here is the equation which defines the locus $\mathcal{L}_{3}$ of fields $K$ with $e_{3}(K) \geq 1$.

$$
C_{8} J_{10}^{8}+C_{7} J_{10}^{7}+\cdots+C_{1} J_{10}+C_{0}=0
$$

where $C_{0}, \ldots, C_{8}$ are

$$
\begin{aligned}
C_{8} & =2^{4} \cdot 3^{31 \cdot} 5^{5} \cdot 19^{10} \cdot 29^{5} \\
C_{7} & =2^{4} \cdot 3^{27} \cdot 19^{5}\left(-194894640029511 J_{2}^{5}-55588661819356000 J_{4}^{2} J_{2}-12239149540657725 J_{2}^{3} J_{4}\right. \\
+ & \left.223103526505680000 J_{4} J_{6}+40811702108053500 J_{2}^{2} J_{6}\right)
\end{aligned}
$$

$C_{6}=2^{2} \cdot 3^{21}\left(-35802284468757765858432 J_{4}^{5}-1756270399106587730391 J_{4}^{2} J_{2}^{6}-28638991859523006654 J_{4} J_{2}^{8}\right.$ $-84091225203760159441286 J_{4}^{3} J_{2}^{4}+400895959391006953561032 J_{4}^{4} J_{2}^{2}-61773685738999443 J_{2}^{1} 0$

$-3673201396072259603756160 J_{4}^{3} J_{2} J_{6}+7879491755218264984387200 J_{4}^{2} J_{6}^{2}+15251447355608658629952 J_{2}^{5} J_{4} J_{6}$ $+1179903008384844066250272 J_{2}^{3} J_{4}^{2} J_{6}-5566672398589809889658760 J_{2}^{2} J_{4} J_{6}^{2}+112024289372554183680 J_{2}^{7} J_{6}$ $\left.-32116769409722716182888 J_{2}^{4} J_{6}^{2}+8512171877754962249155200 J_{2} J_{6}^{3}\right)$ 
$C_{5}=2^{2} \cdot 3^{19}\left(-12630004382695462653 J_{4}^{4} J_{2}^{7}+320839252764287362560 J_{4}^{7} J_{2}-1876069272397136886448 J_{4}^{6} J_{2}^{3}\right.$ $+606742866220456356580 J_{4}^{5} J_{2}^{5}-124173485719052715 J_{4}^{3} J_{2}^{9}+22241034512101438944000 J_{4}^{5} J_{2}^{2} J_{6}$

- $88546736703938826304512 J_{4}^{4} J_{2} J_{6}^{2}-10712078926420753449984 J_{2}^{4} J_{4}^{4} J_{6}+68904635323303664511264 J_{2}^{3} J_{4}^{3} J_{6}^{2}$

- $192353895694677164016384 J_{2}^{2} J_{4}^{2} J_{6}^{3}+197449733923926905783808 J_{2} J_{4} J_{6}^{4}-1916173047371645223936 J_{4}^{6} J_{6}$

$+116211018774997425051648 J_{4}^{3} J_{6}^{3}-132143597697786172416 J_{6}^{5}+1361403542457288 J_{2}^{1} 0 J_{4} J_{6}$

$-5005765118740492656 J_{2}^{7} J_{4} J_{6}^{2}+232819061639483430720 J_{2}^{6} J_{4}^{3} J_{6}-1576319894694585178452 J_{2}^{5} J_{4}^{2} J_{6}^{2}$

$+4655239459208764553088 J_{2}^{4} J_{4} J_{6}^{3}-226900590409548 J_{2}^{11} J_{4}^{2}-2042105313685932 J_{2}^{9} J_{6}^{2}+6261632755967872800 J_{2}^{6} J_{6}^{3}$

$\left.-5065734796478275576176 J_{2}^{3} J_{6}^{4}+1352318109350828796 J_{2}^{8} J_{4}^{2} J_{6}\right)$

$C_{4}=3^{15}\left(1417825317153277312 J_{4}^{9} J_{2}^{2}+2391308818408811717 J_{4}^{6} J_{2}^{8}+718590303030600 J_{2}^{10} J_{4}^{5}\right.$

$-638760745337170544640 J_{4} J_{6}^{6}+440759275303802880 J_{4}^{10}-8118717280771686540192 J_{4}^{5} J_{2}^{4} J_{6}^{2}$

$+42668434906863398019072 J_{4}^{2} J_{2} J_{6}^{5}-57054664814020640574336 J_{4}^{3} J_{2}^{2} J_{6}^{4}+30546774740158581676032 J_{4}^{4} J_{2}^{3} J_{6}^{3}$

$+8601814215123831275904 J_{4}^{6} J_{2}^{2} J_{6}^{2}-1449562700682195916800 J_{4}^{7} J_{2}^{3} J_{6}+1067928354124249303104 J_{4}^{6} J_{2}^{5} J_{6}$

$-10443896263024316301312 J_{2}^{3} J_{4} J_{6}^{5}+7247970315150439028112 J_{2}^{4} J_{4}^{2} J_{6}^{4}-21769241176751736619008 J_{4}^{5} J_{2} J_{6}^{3}$

$-2640201919999154595648 J_{2}^{5} J_{4}^{3} J_{6}^{3}-55893562424445261312 J_{2}^{7} J_{4}^{5} J_{6}+531409635241191119304 J_{2}^{6} J_{4}^{4} J_{6}^{2}$

$-1012614205133520 J_{2}^{8} J_{6}^{4}-2454855015326199552 J_{2}^{5} J_{6}^{5}-12501409939920 J_{2}^{12} J_{4}^{4}-675076136755680 J_{2}^{10} J_{4}^{2} J_{6}^{2}$

$-33390518666828400 J_{2}^{9} J_{4}^{4} J_{6}+3188363568027498432 J_{2}^{6} J_{4} J_{6}^{4}-1569001498547402304 J_{2}^{7} J_{4}^{2} J_{6}^{3}$

$-275375222428239820800 J_{4}^{7} J_{6}^{2}+19809849095518050330624 J_{4}^{4} J_{6}^{4}+6179516061983740183680 J_{2}^{2} J_{6}^{6}$

$+150016919279040 J_{2}^{11} J_{4}^{3} J_{6}+87799481406335621136 J_{4}^{8} J_{2}^{4}+1350152273511360 J_{2}^{9} J_{4} J_{6}^{3}$

$\left.-55496611186132800648 J_{4}^{7} J_{2}^{6}+39911809855842557952 J_{4}^{8} J_{2} J_{6}+353362680242481096 J_{2}^{8} J_{4}^{3} J_{6}^{2}\right)$

$C_{3}=2^{4} \cdot 3^{12}\left(-19225816442103600 J_{4}^{10} J_{2}^{5}+6433952690394144 J_{2}^{4} J_{6}^{7}-2917203075615 J_{2}^{11} J_{4}^{7}\right.$

$+62951605613640 J_{2}^{10} J_{4}^{6} J_{6}+7900854051362368 J_{4}^{11} J_{2}^{3}-873165547551982 J_{2}^{9} J_{4}^{8}+13234982161044480 J_{4}^{12} J_{2}$

$+4077902864550187008 J_{4}^{5} J_{6}^{5}+7506792545698293 J_{4}^{9} J_{2}^{7}-55019014994202624 J_{4}^{11} J_{6}-3415519987075510272 J_{4}^{2} J_{6}^{7}$

$-932605137272623104 J_{4}^{8} J_{6}^{3}-6607177263254292480 J_{2} J_{6}^{8}-1394785406520 J_{2}^{7} J_{6}^{6}-1913285880 J_{2}^{13} J_{4}^{6}$

$-258293593800 J_{2}^{11} J_{4}^{4} J_{6}^{2}-1976299597616301504 J_{4}^{8} J_{2}^{3} J_{6}^{2}+1337598192058041744 J_{4}^{7} J_{2}^{5} J_{6}^{2}$

$-2324642344200 J_{2}^{9} J_{4}^{2} J_{6}^{4}+2789570813040 J_{2}^{8} J_{4} J_{6}^{5}-243015467955111198 J_{2}^{7} J_{4}^{6} J_{6}^{2}+22136761801348668 J_{2}^{8} J_{4}^{7} J_{6}$

$-155463896437263612 J_{4}^{8} J_{2}^{6} J_{6}+16101033796183004352 J_{4}^{5} J_{2}^{3} J_{6}^{4}+16367298631796450304 J_{4}^{3} J_{2} J_{6}^{6}+8433152 J_{4}^{7} J_{2}^{2} J_{6}^{3}$

$-8254965178021469184 J_{4}^{6} J_{2} J_{6}^{4}+34439145840 J_{2}^{12} J_{4}^{5} J_{6}-576988130682378 J_{2}^{9} J_{4}^{5} J_{6}^{2}+2912934238489260 J_{2}^{8} J_{4}^{4} J_{6}^{3}$

$-8749875412454175 J_{2}^{7} J_{4}^{3} J_{6}^{4}+15637511592200340 J_{2}^{6} J_{4}^{2} J_{6}^{5}-127105068829245696 J_{4}^{10} J_{2}^{2} J_{6}+614908581517421568 J_{4}^{9} J_{2} J_{6}^{2}$

$-23374419431360207616 J_{4}^{4} J_{2}^{2} J_{6}^{5}+1508868948605946984 J_{2}^{6} J_{4}^{5} J_{6}^{3}-5795040294470623824 J_{2}^{5} J_{4}^{4} J_{6}^{4}$

$+14094983896511630112 J_{2}^{4} J_{4}^{3} J_{6}^{5}+1033174375200 J_{2}^{10} J_{4}^{3} J_{6}^{3}+314069798204069472 J_{4}^{9} J_{2}^{4} J_{6}-61501104 J_{4}^{6} J_{2}^{4} J_{6}^{3}$

$\left.-21194163080222025024 J_{2}^{3} J_{4}^{2} J_{6}^{6}+18002402119176332544 J_{2}^{2} J_{4} J_{6}^{7}-15392091937240080 J_{2}^{5} J_{4} J_{6}^{6}\right)$

$C_{2}=2^{5} \cdot 3^{8}\left(-159732958548480 J_{4}^{13} J_{2} J_{6}-27945192968593920 J_{2} J_{4} J_{6}^{9}+238596124150086 J_{4}^{10} J_{2}^{7} J_{6}+3224288 J_{2}^{3} J_{6}^{9}\right.$

$-36311136215244 J_{2}^{9} J_{4}^{9} J_{6}-996173640 J_{2}^{12} J_{4}^{6} J_{6}^{2}+5977041840 J_{2}^{11} J_{4}^{5} J_{6}^{3}-22413906900 J_{2}^{10} J_{4}^{4} J_{6}^{4}-37501414009508 J_{4}^{13} J_{2}^{4}$

$+14210312049697149 J_{2}^{6} J_{4}^{6} J_{6}^{4}+86354918885580768 J_{2}^{4} J_{4}^{4} J_{6}^{6}-111444977082978432 J_{2}^{3} J_{4}^{3} J_{6}^{7}+27908893977856 J_{4}^{14} J_{2}^{2}$

$+83768141083825152 J_{2}^{2} J_{4}^{2} J_{6}^{8}-42942980968765488 J_{2}^{5} J_{4}^{5} J_{6}^{5}-10736445647473 J_{4}^{11} J_{2}^{8}+61746352553318400 J_{4}^{4} J_{2} J_{6}^{7}$

$+410958880454688 J_{4}^{12} J_{2}^{3} J_{6}-14059252057660416 J_{4}^{3} J_{6}^{8}-1643659809866496 J_{4}^{11} J_{2}^{2} J_{6}^{2}+441832778741790 J_{2}^{8} J_{4}^{8} J_{6}^{2}$

$-3128599551108636 J_{2}^{7} J_{4}^{7} J_{6}^{3}+2815950495430656 J_{4}^{10} J_{2} J_{6}^{3}+12291244885171152 J_{4}^{8} J_{2}^{5} J_{6}^{3}+1579225145 J_{2}^{12} J_{4}^{9}$

$-2286353789913249 J_{4}^{9} J_{2}^{6} J_{6}^{2}-40300476525629352 J_{4}^{7} J_{2}^{4} J_{6}^{4}+81707043798929088 J_{4}^{6} J_{2}^{3} J_{6}^{5}-25936092270 J_{2}^{6} J_{6}^{8}$

$-98257765274489088 J_{4}^{5} J_{2}^{2} J_{6}^{6}+3318887207480832 J_{4}^{10} J_{2}^{4} J_{6}^{2}-478511899451856 J_{4}^{11} J_{2}^{5} J_{6}+28476287051677 J_{4}^{1} 2 J_{2}^{6}$

$-12153253649302656 J_{4}^{9} J_{2}^{3} J_{6}^{3}+24757975700165376 J_{4}^{8} J_{2}^{2} J_{6}^{4}-26570902457981952 J_{4}^{7} J_{2} J_{6}^{5}-6755065089024 J_{4}^{15}$

$+10883911680 J_{6}^{10}+53793376560 J_{2}^{9} J_{4}^{3} J_{6}^{5}-80690064840 J_{2}^{8} J_{4}^{2} J_{6}^{6}+69162912720 J_{2}^{7} J_{4} J_{6}^{7}+94873680 J_{2}^{13} J_{4}^{7} J_{6}$

$+227109129291 J_{2}^{10} J_{4}^{7} J_{6}^{2}-628213747356 J_{2}^{9} J_{4}^{6} J_{6}^{3}-1389130574661 J_{2}^{8} J_{4}^{5} J_{6}^{4}+16465793988870 J_{2}^{7} J_{4}^{4} J_{6}^{5}$

$-56794191944715 J_{2}^{6} J_{4}^{3} J_{6}^{6}+102713329135152 J_{2}^{5} J_{4}^{2} J_{6}^{7}-98529746457492 J_{2}^{4} J_{4} J_{6}^{8}-30650938650 J_{2}^{11} J_{4}^{8} J_{6}$

$-1716480768 J_{4}^{9} J_{6}^{4}+11718053954519040 J_{4}^{6} J_{6}^{6}+220752428322816 J_{4}^{12} J_{6}^{2}+1322792799725 J_{2}^{10} J_{4}^{10}-3953070 J_{2}^{14} J_{4}^{8}$ )

$C_{1}=-2^{8} \cdot 3^{5}\left(61736960 J_{4}^{8} J_{2}-182135808 J_{4}^{7} J_{6}+16021872 J_{4}^{7} J_{2}^{3}-211022400 J_{4}^{6} J_{2}^{2} J_{6}-26594919 J_{4}^{6} J_{2}^{5}\right.$

$+899159040 J_{4}^{5} J_{2} J_{6}^{2}+330458928 J_{4}^{5} J_{2}^{4} J_{6}-215198 J_{2}^{7} J_{4}^{5}-1227405312 J_{4}^{4} J_{6}^{3}-1535734368 J_{4}^{4} J_{2}^{3} J_{6}^{2}$

$+2930532 J_{4}^{4} J_{2}^{6} J_{6}-363 J_{2}^{9} J_{4}^{4}+3162070656 J_{4}^{3} J_{2}^{2} J_{6}^{3}-16471998 J_{4}^{3} J_{2}^{5} J_{6}^{2}+4356 J_{4}^{3} J_{2}^{8} J_{6}-19602 J_{4}^{2} J_{2}^{7} J_{6}^{2}$ 


$$
\begin{aligned}
& -2433162240 J_{4}^{2} J_{2} J_{6}^{4}+47961936 J_{4}^{2} J_{2}^{4} J_{6}^{3}+39204 J_{4} J_{2}^{6} J_{6}^{3}-72369936 J_{4} J_{2}^{3} J_{6}^{4}+746496 J_{4} J_{6}^{5}-29403 J_{2}^{5} J_{6}^{4} \\
& \left.+45116352 J_{2}^{2} J_{6}^{5}\right)\left(J_{4}^{3}-J_{2}^{2} J_{4}^{2}+6 J_{2} J_{6} J_{4}-9 J_{6}^{2}\right)^{3} \\
& \quad C_{0}=2^{8}\left(768 J_{4}^{2}-416 J_{4} J_{2}^{2}-J_{2}^{4}+1536 J_{2} J_{6}\right)\left(J_{4}^{3}-J_{2}^{2} J_{4}^{2}+6 J_{2} J_{6} J_{4}-9 J_{6}^{2}\right)^{6}
\end{aligned}
$$

\section{Appendix B:}

The equation of the branch locus of the map

$$
\begin{gathered}
\theta: k^{2} \backslash\{\Delta=0\} \rightarrow \mathcal{L}_{3} \\
(u, v) \rightarrow\left(i_{1}, i_{2}, i_{3}\right)
\end{gathered}
$$

(34)

$$
\begin{aligned}
& 3507505273398025 i_{1}^{12}-4880484817793862073548480 i_{1}^{11}+192\left(11302504938388489628125 i_{2}\right. \\
& +346452039237689650581192) i_{1}^{10}-4\left(89439905046278964319358976+60307157046030532997225 i_{2}^{2}\right. \\
& \left.-4188981066069113234648640 i_{2}\right) i_{1}^{9}+192\left(4924672355809568004885504+33588887753890413143515 i_{2}^{2}\right. \\
& \left.-89290373874540245356608 i_{2}\right) i_{1}^{8}-192\left(6360139591235383381327872+593587805135845078438632 i_{2}^{2}\right. \\
& \left.+849397678885114696829952 i_{2}-15537701670163340329775 i_{2}^{3}\right) i_{1}^{7}+2\left(406606074742962841916289024 i_{2}^{2}\right. \\
& -27711519511099200420652160 i_{2}^{3}+305526006347596356290347008+183049808606955774794075 i_{2}^{4} \\
& \left.+255320765313220782576893952 i_{2}\right) i_{1}^{6}-192 i_{2}\left(13654023711946692280289280 i_{2}+60377228453350507376315 i_{2}^{3}\right. \\
& \left.-1464350022771442997265792 i_{2}^{2}+2245754530466537929703424\right) i_{1}^{5}+192 i_{2}^{2}(19906166147692916436664320 \\
& \left.+524465846991778455117432 i_{2}^{2}-2333360138590692031481856 i_{2}-10694743521252049023175 i_{2}^{3}\right) i_{1}^{4} \\
& +20 i_{2}^{2}\left(2535928320048654288481984 i_{2}^{3}-5763664926963183845376000 i_{2}-28262691566657845506883584 i_{2}^{2}\right. \\
& \left.-99413657833844087193600000-9280304409903257402325 i_{2}^{4}\right) i_{1}^{3}-192 i_{2}^{3}\left(1807602326421361731982656 i_{2}^{2}\right. \\
& \left.-76401752022567738591625 i_{2}^{3}-2867627019656613888000000-9918952715424066995911680 i_{2}\right) i_{1}^{2} \\
& +1728 i_{2}^{4}\left(1262371230708245434125 i_{2}^{3}-44970318919363276752280 i_{2}^{2}-1752882366993587712000000\right. \\
& \left.+506870449554602235884544 i_{2}\right) i_{1}+i_{2}^{4}\left(127385395640432909375625 i_{2}^{4}-6059765135286837968377600 i_{2}^{3}\right. \\
& \left.-717148324858373259264000000 i_{2}+102838387547772598641352704 i_{2}^{2}+1617386925920256 \cdot 10^{12}\right)=0
\end{aligned}
$$

\section{REFERENCES}

[1] G. FreY, On elliptic curves with isomorphic torsion structures and corresponding curves of genus 2. Elliptic curves, modular forms, and Fermat's last theorem (Hong Kong, 1993), 79-98, Ser. Number Theory, I, Internat. Press, Cambridge, MA, (1995).

[2] G. Frey and E. Kani, Curves of genus 2 covering elliptic curves and an arithmetic application. Arithmetic algebraic geometry (Texel, 1989), 153-176, Progr. Math., 89, Birkhäuser Boston, Boston, MA, (1991).

[3] H. Lange, Über die Modulvarietät der Kurven vom Geschlecht 2. J. Reine Angew. Math., 281, 80-96, 1976.

[4] E, Kani And W. Schanz, Diagonal quotient surfaces. Manuscripta Math. 93 (1997), no. 1, $67-108$.

[5] E. KAnI, The number of curves of genus two with elliptic differentials. J. Reine Angew. Math. 485 (1997), 93-121.

[6] A. Krazer, Lehrbuch der Thetafunctionen, Chelsea, New York, (1970).

[7] V. Krishnamoorthy, Invariants of genus 2 curves, PhD thesis, University of Florida, 2001.

[8] M. R. Kunn, Curves of genus 2 with split Jacobian. Trans. Amer. Math. Soc 307 (1988), 41-49

[9] J. Igusa, Arithmetic Variety Moduli for genus 2. Ann. of Math. (2), 72, 612-649, (1960).

[10] Maple 6, Waterloo Maple Inc., 2000.

[11] T. Shaska, Genus 2 Curves With (N,N) Decomposable Jacobians, Jour. Symb. Comp., Vol 31 , no. 5, pg. 603-617, 2001. 
[12] T. Shaska, Curves of Genus Two Covering Elliptic Curves, PhD thesis, University of Florida, 2001.

[13] T. Shaska And H. VÖlklein, Elliptic Subfields and automorphisms of genus 2 function fields, to appear in: Proceeding of the Conference on Algebra and Algebraic Geometry with Applications: The celebration of the seventieth birthday of Professor S.S. Abhyankar, SpringerVerlag, 2000.

[14] G. Tamme, Ein Satz über hyperelliptische Funktionenkörper. J. Reine Angew. Math. 257 (1972), 217-220.

[15] H. VöLkLein, Groups as Galois Groups - an Introduction, Cambr. Studies in Adv. Math. 53, Cambridge Univ. Press 1996.

Department of Mathematics, University of California at Irvine, Irvine, CA 92697.

E-mail address: tshaska@math.uci.edu 